\title{
The Quasi-Harmonic Approximation and a Generalized Grüneisen Equation of State
}

\author{
Frederick I. Mopsik
}

Institute for Materials Research, National Bureau of Standards, Washington, D.C. 20234

(February 8, 1973)

\begin{abstract}
The validity of the Grüneisen equation of state for a solid having a continuous distribution of frequencies is investigated. It is shown that one cannot generally replace $\partial S / \partial V$ with the heat capacity multiplied by appropriate Grüneisen constants. A model frequency distribution is used to show the difference that can arise.
\end{abstract}

Key words: Crystal equation of state; frequency distribution; Grüneisen constant; Grüneisen equation of state; quasi-harmonic approximation; thermal expansion.

\section{Introduction}

The Grüneisen equation of state has been widely used to discuss the thermal properties of solids. While it is reasonably successful with simple monatomic solids, it has been found to be less adequate with polyatomic solids and the need to expand its definition has been well established.

The principal idea in the modification of the simple Grüneisen equation has been the recognition that the frequency spectrum of a solid is not characterized by just a single frequency. Rather, many frequencies are involved and not all of them shift with volume in the same way. Thus, Blackman $[1,2]^{1}$ replaces the simple Grüneisen equation

with

$$
\alpha=\gamma C_{v} / B_{T} V
$$

$$
\alpha=\sum_{i} \gamma_{i} C_{v i} / B_{T} V
$$

where the Grüneisen constant is defined as $\gamma_{i}=$ $-d \ln \nu_{i} / d \ln V, B_{T}=-V\left[\frac{\partial p}{\partial V}\right]_{T}, C_{v i}$ is the specific heat for frequency $v_{i}, P$ is the pressure and $V$ is the molar volume. Blackman does go further and allows the summation to become an integral where now

$$
\alpha=\int \gamma(\nu) C_{v}(\nu) \rho(\nu) d \nu / B_{T} V
$$

where $\rho(\nu)$ is the spectral density for frequency $\nu$. However, eq (2) directly implies only an integral over the index $i$ (or the wave vector q) and it should be asked

\footnotetext{
${ }^{1}$ Figures in brackets indicate the literature references at the end of this paper.
}

as to whether eq (3) is a proper integral. If the index is one-dimensional and if there is a unique value of $\nu$ associated with every value of the index, then the transformation will go through to eq (3). If the index is an $n$ dimensional wave vector, then there will be an $n-1$ dimensional surface of constant $\nu$ and only if for all the points on the surface is $\gamma$ a constant, will eq (3) result. Obvious cases where this will hold, such as having only one force constant or having all the force constants have the same logarithmic derivative actually lead to eq (1) rather than (3) since $\gamma$ is a constant for all $\nu$. If $\nu$ involves sums of force constants multiplied by functions of the wave vector, and if each force constant has a different logarithmic derivative then the conditions for (3) are clearly not satisfied and the question of a proper replacement for (3) arises.

In the course of previous work $[3,4]$ on soft crystals, it became apparent that eq (1) was quite inadequate to reproduce the thermal expansion and yet it was felt that the quasi-harmonic approximation inherent in the Grüneisen equation still might be adequate. This consideration has led to the extension of simple Grüneisen theory described below that in proper limits reduces to eqs (1) and (2) and yet is more widely applicable.

\section{Theory}

The derivation of the main equation is straightforward and is within the scope of general quasiharmonic theory. In the following equations we will consider a solid with $n$ dispersion curves characterizing its frequency spectrum with no attempt to distinguish between acoustic or optical branch values unless indicated. Furthermore, only one branch will 
be considered at a time since the general result will be a simple summation over like terms for each branch.

From thermodynamics,

$$
\alpha B_{T}=\frac{\partial^{2} A}{\partial T \partial V}
$$

Using quasi-harmonic lattice dynamics, one may write the Helmholtz free energy as

$$
A=\int_{\nu_{1}}^{\nu 2} F(\nu) \rho(\nu) d \nu
$$

where $F(\nu)$ is the Einstein free energy function [5] for frequency $\nu$ and $\rho(\nu)$ is the frequency density function. In order to ensure that all functions are properly single valued and smooth, the integral may be restricted to the frequency interval $\left[\nu_{1}, \nu_{2}\right]$ and to only one branch of the frequency spectrum. When this is done $A$ will simply be the sum of integrals like that of eq (5).

Using the quasi-harmonic approximation, $\rho(\nu)$ is a function of volume only and so are $\nu_{1}$ and $\nu_{2}$ while $F(\nu)$ is a function of temperature but not volume. Therefore,

$$
\alpha B_{T}=-\frac{\partial}{\partial V} \int_{\nu_{1}}^{\nu 2} S(\nu) \rho(\nu) d \nu
$$

where $S(\nu)$ is the Einstein entropy function and the indicated temperature differentiation has been carried out.

The main point of this paper is the interesting observation that eq (6) is as far as one can go and keep the full generality of the quasi-harmonic approximation. It should be noted that no further assumptions have been added and the mathematics has been quite rigorous.

The question arises as to how a Grüneisen equation can be derived from eq (6). The answer is that if $A$ is a function of $\theta / T$ and $\theta$ which is equal to $h \nu / k$ is volume dependent only, then one can reduce eq (6) to eq (1) as has been shown by Kittel [6]. This assumption also validates eq (2) since a summation of terms in $\theta_{i} / T$ also can be carried out. Unfortunately, eq (3) cannot be so obtained. This can be seen by applying the Leibnitz rule for the differentiation of a definite integral to eq (6) to obtain

$$
\begin{array}{rl}
\alpha B_{T}=-\int_{\nu_{1}}^{\nu_{2}} & S(\nu) \frac{\partial \rho(\nu)}{\partial V} d \nu \\
& -S\left(\nu_{2}\right) \rho\left(\nu_{2}\right) \frac{\partial \nu_{2}}{\partial V}+S\left(\nu_{1}\right) \rho\left(\nu_{1}\right) \frac{\partial \nu_{1}}{\partial V}
\end{array}
$$

Equation (7) is now seen not only to have a different integral but to include two extra terms that cannot be related in any manner to eq (3) since they involve entropy terms that will not reduce to heat capacity terms or will not cancel in general. Therefore, it is eq (7) and not eq (3) that is the proper expression to use for a continuous distribution of frequencies.

A Grüneisen type analysis can be obtained if $\rho(\nu)$ is $\rho\left(\nu ; \nu_{1}, \nu_{2}, \ldots \nu_{m}\right)$ where $\rho$ is a function of volume only through the parameters $\nu_{i}$, which are expressed as frequencies. Then eq (7) can be written

$$
\begin{aligned}
\alpha B_{T} V= & \sum_{i} \gamma_{i} \nu_{i} \int_{\nu_{1}}^{\nu_{2}} S(\nu) \frac{\partial \rho(\nu)}{\partial \nu_{i}} d \nu \\
& +\gamma_{2} \nu_{2} S\left(\nu_{2}\right) \rho\left(\nu_{2}\right)-\gamma_{1} \nu_{1} S\left(\nu_{1}\right) \rho\left(\nu_{1}\right) .
\end{aligned}
$$

Note that unlike eq (3) there is no $\gamma(\nu)$ appearing in (8). This is a result of the use of a definite integral; the variable $\nu$ is a variable of integration and merely serves to define a function of the other variables. Therefore, it is not available for formation of a Grüneisen parameter and there is no rigorous physical way to define any $\gamma(\nu)$ when using only a $\rho(\nu)$. It is only when the frequency spectrum changes with volume with respect to a finite number of critical frequencies that a Grüneisen parameter analysis can be used and then only in the form of eq (8). Again it should be noted that in the special case that a Debye or Einstein distribution is used, eq (8) will reduce to eq (1).

\section{Application}

While eqs (7) and (8) look more forbidding than the usual extensions to Grüneisen theory its application can be straightforward since, if the entropy as a function of state is known, only differentiations are required. This is illustrated quite well by the previous work on linear carbon chains [3, 4].

In that work an approximation scheme was used to generate the frequency spectrum of a finite linear chain. Each branch of the spectrum was assumed to be such that the frequency density was proportional to the frequency between the free chain frequency and a higher frequency that was the root of the sum of the squares of the free chain frequency and the highest lattice frequency corresponding to the entire chain moving as a unit. Only the latter frequency was allowed to vary with volume as only it depended on interchain force constants. This is in analogy with a linear diatomic chain of equal masses and alternating force constants, where for small changes in the weaker force constant there is little change in shape of the dispersion curves and the limits on the frequencies correspond to the assumptions made for the finite chains.

This model allowed an easy application of the method of this paper with rather good success. For this model

$$
\rho(\nu)=\frac{2 \nu}{\nu_{2}^{2}-\nu_{1}^{2}}, \quad \nu_{2} \geqslant \nu \geqslant \nu_{1} \text {. }
$$


TABLE 1

\begin{tabular}{c|c|c|c|r|r|r}
\hline \multirow{2}{*}{$\nu_{1} / \nu_{2}$} & \multicolumn{2}{|c|}{.1} & \multicolumn{2}{|c|}{.5} & \multicolumn{2}{c}{.9} \\
\cline { 2 - 7 } & $\frac{1}{\gamma R} \frac{\partial S}{\partial V}$ & $C_{v} / R$ & $\frac{1}{\gamma R} \frac{\partial S}{\partial V}$ & $C_{v} / R$ & $\frac{1}{\gamma R} \frac{\partial S}{\partial V}$ & $C_{v} / R$ \\
\hline 0.05 & 0.0187 & 0.02781 & 0.0001 & 0.0004 & 0.0000 & 0.0000 \\
.1 & .1149 & .1348 & .0189 & .0410 & .0032 & .0069 \\
.2 & .4142 & .4437 & .2108 & .3371 & .1014 & .1987 \\
.4 & .7486 & .7831 & .5077 & .7343 & .3362 & .6373 \\
.6 & .8573 & .8931 & .6125 & .8683 & .4330 & .8145 \\
.8 & .9013 & .9375 & .6557 & .9229 & .4745 & .8901 \\
1.0 & .9228 & .9593 & .6770 & .9497 & .4953 & .9279 \\
10.0 & .9627 & .9996 & .7168 & .9995 & .5347 & .9992 \\
\hline
\end{tabular}

In applying eq (8) to (9), $\nu_{1}$ was assumed to be independent of frequency so that

$$
\begin{aligned}
& \alpha \frac{B_{T} V}{R \gamma_{2}}=\frac{\nu_{2}}{\left[1-\left(\nu_{1} / \nu_{2}\right)^{2}\right]^{2}}\left\{3\left[D\left(\nu_{2}\right)-\left(\frac{\nu_{1}}{\nu_{2}}\right)^{2} D\left(\nu_{1}\right)\right]\right. \\
& \left.-2\left(\frac{\nu_{1}}{\nu_{2}}\right)^{2}\left[F\left(\nu_{2}\right)-F\left(\nu_{1}\right)\right]-2\left[1-\left(\frac{\nu_{1}}{\nu_{2}}\right)^{2}\right] E\left(\nu_{2}\right)\right\}
\end{aligned}
$$

where $D(\nu), E(\nu)$ and $F(\nu)$ are the two dimensional Debye function, the Einstein internal energy function and the Einstein free energy function respectively and where $R$ is the gas constant. If $\nu_{1}$ approaches zero, eq (10) is seen to become the ordinary Grüneisen relation with the heat capacity being that for a two-dimensional Debye solid. However, as $\nu_{1}$ becomes greater than zero, departures will occur. This is.illustrated in table 1 where both the heat capacity and $\partial S / \gamma \partial V$ are compared for a few different frequency intervals. Note the different assymptotic limits for the two functions.

\section{Conclusion}

From the above considerations, it can be seen that the extension of the quasi-harmonic approximation and Grüneisen analysis to complex solids is not as simple as has been generally postulated. One must introduce Grüneisen parameters with care and they can be defined only on the more restrictive condition that the frequency spectrum is defined, as a function of volume, by a discrete number of critical frequencies. Even for this case one cannot use a simple Grüneisen eq (1) but must replace the heat capacity term by a proper derivative of the entropy function as in eq (8).

If this is done, however, it is then possible to write explicit forms for the equation of state that introduce no further assumptions into the model.

This is a great benefit in investigating model calculations. Also, it can be seen that empirical checks of quasi-harmonic approximation in terms of thermal expansion are more involved than generally realized since the quantity $(\partial S / \partial V)_{T}$ is not one that is readily available experimentally and yet is the quantity that must be used.

\section{References}

[1] Blackman, M., Proc. Phys. Soc. (London) B70, 827 (1957).

[2] Blackman, M., Proc. Phys. Soc. (London) B74, 17 (1959).

[3] Broadhurst, M. G., and Mopsik, F. I., J. Chem. Phys. 52, 3634 (1970).

[4] Broadhurst, M. G., and Mopsik, F. I., J. Chem. Phys., 54, 423y (1971).

[5] The definition of the Einstein and Debye functions used here are to be found in Handbook of Mathematical Functions, M. Abramowitz and I. A. Stegun, eds., National Bureau of Standards, Applied Mathematics Series, 55, June, 1964.

[6] Kittel, C., Introduction to Solid State Physics, (John Wiley \& Sons, Inc., New York), pp. 79-81.

(Paper 77A4-780) 